\title{
ANESTESIA PARA CIRUGÍA FETAL FETOSCÓPICA: SECUENCIA DE PERFUSIÓN ARTERIAL REVERSA EN EMBARAZO GEMELAR
}

\author{
Marjorie Lisseth Calderón Lozano"1, Wilber Ccopa Tito², Nelida Vanessa Gil Malca², Nickholas Aguilar Cristobal ${ }^{4}$, \\ Hector Quispe Carrascal ${ }^{5}$, Renato Moreno Gonzales ${ }^{6}$.
}

\begin{abstract}
RESUMEN
Introducción: En los embarazos gemelares con diagnóstico de Secuencia de perfusión arterial reversa (TRAP), se recomienda realizar el tratamiento intra-útero mediante la fotocoagulación bipolar por fetoscopía, con la finalidad de mejorar el pronóstico de uno de los fetos (feto bomba), pues el otro feto es acardio y/o anencefálico, motivo por el cual es necesaria una anestesia que garantice la seguridad materna-fetal y proporcione las condiciones quirúrgicas idóneas. Objetivo: Describir el manejo anestésico de una paciente con diagnóstico de secuencia TRAP programada para fetoscopía-fotocoagulación bipolar. Métodos y resultados: Mostramos el caso de una primigesta de 21 semanas con diagnóstico de secuencia TRAP, a quien se le realizó una fotocoagulación bipolar por fetoscopía, y cuya técnica anestésica empleada fue CSE (combinada espinal-epidural) más sedación con remifentanilo.Conclusiones: La anestesia regional más la sedación con remifentanilo es una buena opción como técnica anestésica en las cirugías fetales fetoscópicas para fotocoagulación bipolar.
\end{abstract}

Palabras clave: Secuencia TRAP; Embarazo gemelar; Manejo anestésico; Combinada espinal-epidural; Remifentanilo (Fuente DeCS BIREME).

\section{ANESTHESIA FOR FETAL SURGERY FETOSCOPY: SEQUENCE OF REVERSE BLOOD PERFUSION IN TWIN PREGNANCY}

\begin{abstract}
Introduction. In twin pregnancies with a diagnosis of Reversed Arterial Perfusion (TRAP), sequence, is highly recommended to be managed by fetoscopic fetal surgery through bipolar photocoagulation, in order to improve the prognosis of one of the fetuses (pump fetus), in contrast of the other one which is acardiac and / or anencephalic. In this regards anesthesia management has to ensure maternal and fetal safety and provide excellent surgical conditions. Objective. To describe the anesthetic management of a patient diagnosed with TRAP sequence scheduled for bipolar fetoscopic-photocoagulation. Methods and results. We describe the case of a 21 gestational week primiparous patient diagnosed with TRAP sequence, who underwent a fetoscopic bipolar photocoagulation under a CSE (combined spinal epidural) technique and a remifentanil infusion for sedation. Conclusions. Regional anesthesia plus sedation with remifentanil is a good choice as an anesthetic technique in fetoscopic fetal surgery for bipolar photocoagulation.
\end{abstract}

Key words: Reversed Arterial Perfusion (TRAP) sequence; Twin pregnancies; Anesthesia management; Combined spinal epidural; Remifentanil (Source:MeSH NLM).

\section{INTRODUCCIÓN}

Los embarazos gemelares, tienen una incidencia de $2-3 \%$ del total de gestaciones, y casi se ha duplicado en las últimas dos décadas, como resultado del aumento de la edad materna que conlleva al empleo de tecnologías de reproducción asistida ${ }^{1}$.

El momento en que ocurre la división celular influye en la formación posterior: la división temprana, dentro de los primeros tres días después de la fecundación tiene como resultado la formación bicorial y biamniotico; la división entre los tres a nueve días después de la fecundación tiene como resultados la formación monocorial y biamniotica; La división después de unos nueve días: monocorial y monoamniotico ${ }^{2}$.
La secuencia de perfusión arterial reversa (twin reversed arterial perfusion [TRAP]), es una rara complicación exclusiva de las gestaciones múltiples monocoriales. Se caracteriza por la presencia de un feto malformado que tiene un corazón rudimentario o ausente, y no tiene perfusión directa placentaria, sino que ésta le llega a través de una comunicación arterio-arterial (A-A) desde el co-gemelo estructuralmente normal (o feto bomba) y luego doblemente desoxigenada tras perfundir al acardio, retorna al feto bomba por una conexión veno-venosa ( $\mathrm{V}-\mathrm{V})$. La historia natural de la enfermedad es la muerte por falla cardiaca del gemelo bomba en $35-55 \%$ de los casos ${ }^{3,4}$.

El primer caso fue reportado por Benedetti en 1533, se han notificado muy pocos casos en la literatura desde entonces. Actualmente la incidencia es 1 de cada 35.000

\footnotetext{
1-5 Médico Residente de Anestesiología, Analgesia y Reanimación del Instituto Nacional Materno Perinatal.Lima-Perú

6 Médico Asistente de Anestesiología, Analgesia y Reanimación del Instituto Nacional Materno Perinatal. Instructor Latinoamericano Curso SAFE: Anestesia Obstétrica. Lima-Perú.
} 


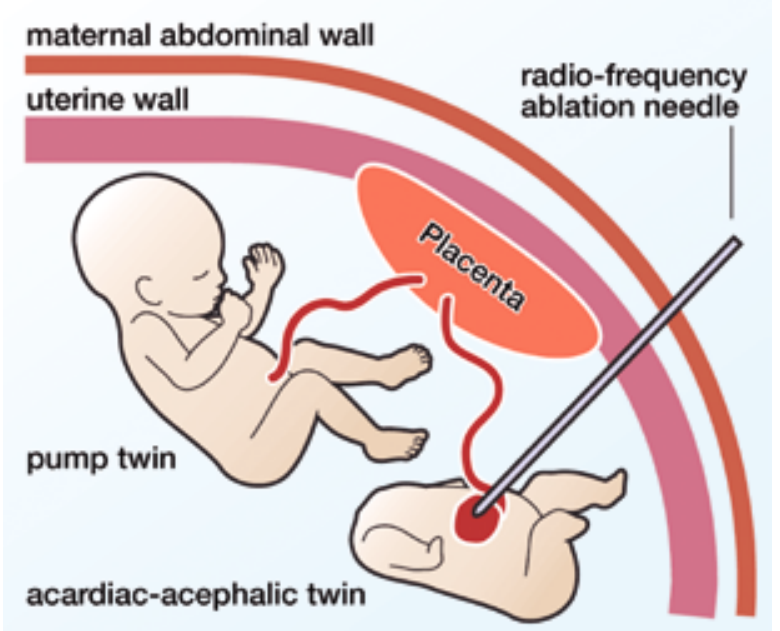

Figura 1. Embarazo gemelar monocoriónico-biamniótico (Secuencia TRAP), se puede visualizar a la izquierda el feto bomba o receptor y a la derecha el feto usualmente acefálico-acardio o donante. Reproducido de UCSF Fetal Treatment Center. http://fetus.ucsf.edu/trap-sequence.

nacimientos, y el riesgo de recurrencia se estima que es $1: 10.000^{3,4}$.

El tratamiento intrauterino puede llevar a cambiar drásticamente el pronóstico y la calidad de vida del feto y de la madre. Para esto se requiere brindar anestesia tanto a la madre como al feto, buscando garantizar las condiciones quirúrgicas adecuadas y evitar efectos deletéreos.

\section{DESCRIPCIÓN DEL CASO}

Primigesta de 24 años de edad, con embarazo gemelar monocoriónico de 21 semanas y diagnóstico de Secuencia TRAP, programada para Fetoscopía y coagulación bipolar. Sin antecedentes personales de importancia y hallazgo ecográfico de feto II acardio. ASA II, Al examen físico; peso: $76 \mathrm{Kg}$; talla: $161 \mathrm{~cm}$; IMC: 29.32; presión arterial (PA), 100/50 mmHg; frecuencia cardiaca (FC), $68 /$ min. Sin predictores de vía aérea difícil, ni hallazgos patológicos cardiopulmonares o neurológicos. Se le explica a la paciente la técnica anestésica y luego de mencionarle los riesgos y beneficios de la estrategia anestésica, se considera realizar una técnica combinada espinal-epidural, para luego administrar una infusión de Remifentanilo para sedación.

Previo a la anestesia, se administró Nifedipino $20 \mathrm{mg}$ VO. Además de $50 \mathrm{mg}$ de Ranitidina i.v., $10 \mathrm{mg}$ de Metoclopramida i.v, Cefazolina $2 \mathrm{~g}$ i.v. Se realizó monitorización ASA básica con preparación de equipo de vía aérea difícil. En posición decúbito lateral izquierda, previa asepsia y antisepsia se ubicó el espacio epidural L3-L4 a $5 \mathrm{~cm}$ de la piel; usando aguja Tuohy $\mathrm{N}^{\circ} 18$ y con técnica de pérdida de resistencia intermitente con aire, se llegó al espacio epidural sin complicaciones. Luego se

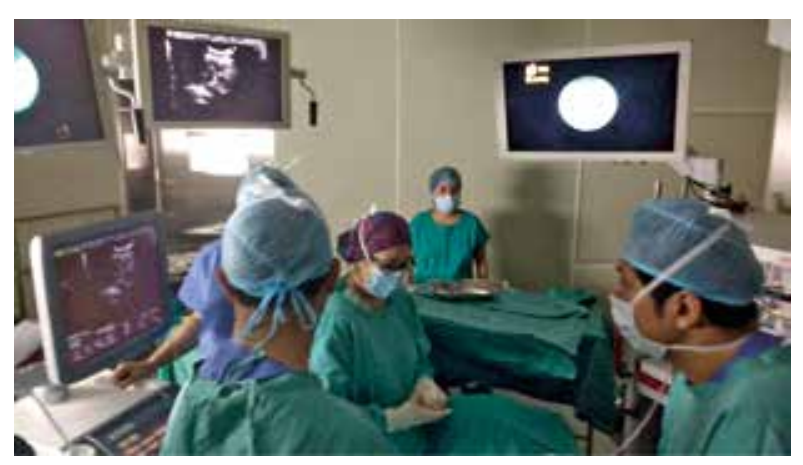

Figura 2. Equipo de cirugía fetal mapeando el borde de la placenta mediante ecografía para posteriormente insertar el trocar de 11 french. Fuente: Los autores.

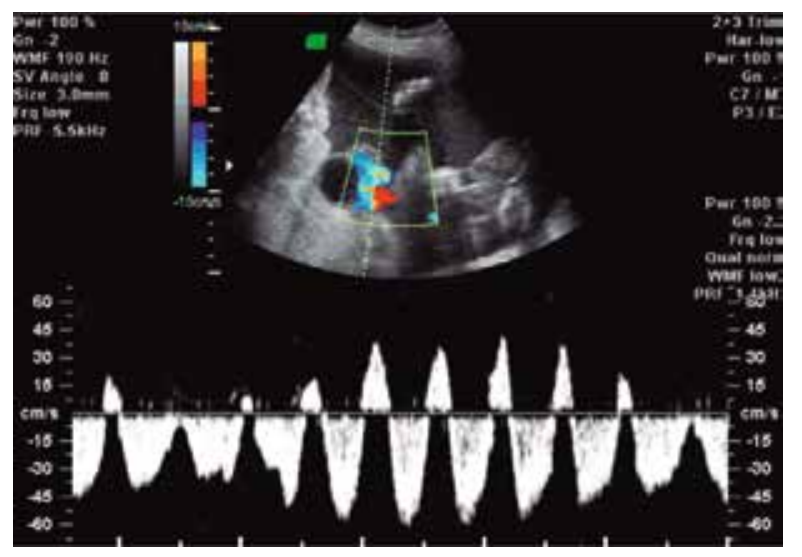

Figura 3. Ultrasonido doppler preformado sobre la arteria umbilical del feto receptor con la presencia de flujo inverso. Reproducida de Mărginean C, Mărginean MO. Rom J Morphol Embryol 2016, 57(1):259-265. The TRAP (twin reversed arterial perfusion) sequence.

procedió a insertar la aguja Whitacre $\mathrm{N}^{\circ} 27$ a través de la aguja Tuohy $\mathrm{N}^{\circ} 18$ y se inyectó bupivacaína hiperbárica $10 \mathrm{mg}$. Finalmente se pasó catéter epidural multiorificio $\mathrm{N}^{\circ} 20$ y se fijó a $10 \mathrm{~cm}$ de la piel. La prueba de aspiración del catéter fue negativa y se decidió no realizar dosis de prueba, se alcanzó un nivel sensorial anestésico hasta T4. Remifentanilo en infusión $(0,05-0,10 \mathrm{ug} / \mathrm{Kg} / \mathrm{min})$ brindó sedación durante el procedimiento y apoyo con máscara con reservorio a $6 \mathrm{~L} / \mathrm{min}$ se administró para garantizar la adecuada oxigenación materna y fetal.

Se inició la cirugía sin incidentes, se mapeó el borde de la placenta mediante ecografía y se insertó un trocar flexible de 11 French, luego se procedió a la inserción de fetoscopio de $3.3 \mathrm{~mm}$, visualizándose ambos gemelos, gemelo I estructuralmente sano y gemelo II acardio, se fotocoaguló el cordón umbilical del gemelo acárdico a 30 wt. El Ultrasonido Doppler confirmó la ausencia de flujo sanguíneo en el gemelo receptor y la función cardiaca normal en el gemelo donante.

Se administró por el catéter epidural una dosis suplementaria de Bupivacaína al $0.5 \%$ de $50 \mathrm{mg}$ a los 100 minutos de la dosis espinal de Bupivacaína, así 
como también Morfina epidural 3mg para manejo de dolor postoperatorio. El intraopeatorio cursó sin interrecurrencias, manteniéndose hemodinámicamente estable durante toda la cirugía, con PA media entre $67-78 \mathrm{mmHg}$, FC materna entre 65-80 lat/minuto, con saturación de $\mathrm{O} 2$ igual o mayor a $98 \%$, ETCO2 entre $30-35 \mathrm{mmHg}$. La administración de cristaloides IV mediante calentador de fluidos ascendió a $800 \mathrm{ml}$. Se procedió a retiro de catéter epidural en recuperación y luego pasó a Hospitalización. La paciente recibió Nifedipino $10 \mathrm{mg}$ VO cada 8 horas y Cefazolina $1 \mathrm{~g}$ EV cada 6 horas durante las siguientes 48 horas. El binomio madre-feto se mantuvo estable y con evolución favorable hasta el alta, que se produjo al tercer día postoperatorio.

\section{DISCUSIÓN}

La anestesia para cirugía fetal envuelve la coordinación entre el equipo quirúrgico y anestesiológico, cada vez más se incrementa el número de cirugías fetales debido al pronto diagnóstico y al entrenamiento del cirujano en este tipo de procedimientos. El diagnóstico de Secuencia TRAP y la ablación de las anastomosis vasculares placentarias mediante laser por fetoscopía son el tratamiento actual de elección ${ }^{5,6}$.

Dentro del manejo anestésico de la cirugía fetoscópica que involucra la manipulación de la placenta y el cordón umbilical, se debe tener en cuenta la anestesia sobre la madre y el feto. La preparación preoperatoria enfocada en la evaluación de la madre con énfasis en sus antecedentes familiares y maternos de problemas anestésicos, evaluación de vía aérea y problemas médicos concurrentes, la profilaxis para prevenir la aspiración, así como una adecuada hidratación y monitoreo básico durante el perioperatorio es importante $7,8,9,10$.

La prevención de la broncoaspiración (ayuno mayor de 8 horas, bloqueantes $\mathrm{H}_{2}$, metoclopramida $10 \mathrm{mg}$ y ondansetrón 4-8 mg); y la disminución del tono vagal y la reducción de las secreciones tráqueobronquiales como el glicopirrolato, no tiene efectos sobre la FCF, al no atravesar la barrera placentaria ${ }^{6}$. La antibiótico profilaxis en cirugía fetal es necesaria para disminuir el riesgo de corioamnioitis y sepsis, que se presentan en menos del $5 \%$ de los casos ${ }^{11}$.

Considerando que la anestesia puede ir desde una anestesia local hasta una anestesia regional (espinal, epidural o espinal/epidural) para la anestesia materna; la técnica dependerá de la complejidad del procedimiento así como su duración. En procedimientos con mínima invasión y de corta duración, como son procedimientos menores a 15 min de duración e inserción de un solo trócar, pueden resolverse con la administración de una sedación materna e infiltración local con lidocaína más adrenalina en los planos. Cuando el procedimiento es mayor en tiempo o es necesario la inserción de más de un trocar, la combinación de un bloqueo neuroaxial, como la espinal, epidural, o combinada espinal-epidural, apoyándose con midazolam más fentanilo o remifentanilo por vía IV, son una excelente elección ${ }^{5-7,10}$.

En esta clase de intervenciones, donde hasta el más ligero movimiento del feto durante la cirugía puede resultar en trauma fetal, hemorragia, compromiso de la circulación umbilical produciendo la muerte materna. Así como la existencia de evidencia acerca de que el feto puede experimentar dolor, y esto repercutir en la supervivencia y neurodesarrollo fetal ${ }^{14}$; El remifentanilo se ha considerado de elección en estudios doble ciego, reportando una adecuada inmovilización fetal, con buena sedación materna y mínima depresión respiratoria, en infusión continua a dosis de $0.1-0.2 \mu \mathrm{g} / \mathrm{kg} / \mathrm{min}^{6,9,12,13,14}$.

El riesgo de que la gestante presente contracciones uterinas con amenaza de parto prematuro, aumenta, siendo el tratamiento tocolítico esencial durante el perioperatorio. Entre los principales agentes tocolíticos usados en este escenario están: antagonistas de los canales de calcio principalmente nifedipino $(10-20 \mathrm{mg})$; B-adrenérgicos; Sulfato de Magnesio (4 a $6 \mathrm{~g}$ por vía IV durante 20 minutos, seguido de una infusión IV de 1 a $2 \mathrm{~g}$ por hora); y antiinflamatorios no esteroideos como la indometacina $50 \mathrm{mg} .6,9,11,13,14$.

En el manejo anestésico se debe tener en cuenta evitar la hipotensión materna, (PAM por encima de $65 \mathrm{mmHg}$ ), evitar la sedación materna excesiva (RASS +1) para evitar así la hipercapnea y mantener EtCO2 alrededor de $30 \mathrm{mmHg}$ (asegurarando una gradiente entre el $\mathrm{PaCO} 2$ materno y fetal). Al ser la asfixia intrauterina el riesgo fetal más importante, es necesario asegurar la estabilidad de la hemodinamia materna, y así un buen flujo sanguíneo uteroplacentario, el uso de vasopresores está descrito ${ }^{11,16}$. Se debe considerar además el manejo de líquidos intraoperatorios restringido $(<1000 \mathrm{~mL})$, para minimizar el riesgo de edema agudo pulmonar asociado con el manejo de la terapia tocolítica ${ }^{12}$.

Un adecuado control del dolor materno perioperatorio es necesario para la prevención de las contracciones uterinas y el parto prematuro; para lo cual, la analgesia postoperatoria se puede mantener con una infusión epidural continua de una solución diluida de anestésico local al $0.1-0.125 \%$ y opioide ${ }^{11}$.

El manejo anestésico de esta paciente fue planeado en función de la fisiología materna y fetal. Se realizó la premedicación convencional para paciente obstétrica, así como la profilaxis antibiótica, montorización estándar, y la administración de oxígeno suplementario. Se optó por una anestesia combinada, debido al tiempo operatorio relativamente largo, por ser ésta una cirugía no realizada frecuentemente en nuestra institución, además que ésta ténica nos permitió manejar la analgesia postoperatorio con Morfina a través del catéter epidural. Finalmente, decidimos usar Remifentanilo para la sedación materna y fetal, por ser éste el opiode de elección para este tipo de procedimientos. 


\section{CONCLUSIONES}

Una adecuada anestesia es fundamental para un óptimo resultado perioperatorio de la madre y el feto o fetos. El anestesiólogo debe conocer y estar preparado para los desafíos que enmarca el progreso de avances tecnológicos que permiten en la actualidad realizar intervenciones quirúrgicas intra-útero. Ésta es la primera presentación de un caso que describe el manejo anestésico para cirugía fetal realizado en Perú. La anestesia regional aunada a la sedación con Remifentanilo, parecen ser la técnica anestésica ideal, creando las condiciones idóneas para la cirugía fetal laparoscópica. Así mismo, la estabilidad hemodinámica, la restricción de fluidos, el uso de tocoliticos y una adecuada analgesia postoperatoria garantizan la seguridad para la madre y el feto.

\section{Responsabilidades éticas}

Protección de personas y animales. Los autores declaran que para esta investigación no se han realizado experimentos en seres humanos ni en animales.

Confidencialidad de los datos. Los autores declaran que han seguido los protocolos de su centro de trabajo sobre la publicación de datos de pacientes.

Derecho a la privacidad y consentimiento informado. Los autores han obtenido el consentimiento informado de los pacientes y/o sujetos referidos en el artículo. Este documento obra en poder del autor de correspondencia.

\section{Financiamiento}

Los autores no recibieron patrocinio para llevar a cabo este artículo.

\section{Conflicto de intereses}

Los autores declaran no tener ningún conflicto de intereses.

\section{Agradecimientos}

Al Dr. Eddisson Quispe Pilco y el Dr. Gunther Vásquez Rojas, Médicos Asistentes del Departamento de Anestesiología, Analgesia y Reanimación del Instituto Nacional Materno Perinatal.
Al Dr. Pedro Saldaña Montes, Jefe del Servicio de Anestesiología, Analgesia y Reanimación del Instituto Nacional Materno Perinatal.

\section{REFERENCIAS BIBLIOGRÁFICAS}

1. Shur N. La genética de hermanamiento: A partir de la división de los huevos que se rompen paradigmas. Am J Med Genet Parte C. Semin Med Genet 2009; 151C: 105-109.

2. Benirschke K, Kim CK. El embarazo múltiple. N Eng J Med. 1973; 288: 1276-84.

3. Shashidhar B, Kumar BN, Sheela R, R Kalyani, Anithae N, Reddy PS. Doble invierte la perfusión (TRAP) secuencia arterial: (Acardius amorfo) de un caso y revisión de la literatura. Int J Biol Med Res. 2012; 3: 1453-55.

4. Fernanda L, Molina FS, Fresneda MD, Padilla MC, Secuencia TRAP: diagnóstico, opciones de tratamiento y experiencia propia. diagn prenat. 2012; 23(4):160-166.

5. Marc Van de Velde Frederik De Buck. Fetal and Maternal Analgesia/Anesthesia for Fetal Procedures. Fetal Diagn Ther 2012; 31: 201-209.

6. Martínez GG. Anestesia para cirugía fetal. Rev Mex Anest 2012; 35: 93-99.

7. Saxena KN, Anesthesia for fetal surgeries. Indial Journal of Anesthesia 2009; 53(5): 554-559.

8. Hans P. Sviggum, Bhavani Shankar Kodali. Maternal Anesthesia for Fetal Surgery Clinics in Perinatology. 2013; 40(3): 413-427.

9. Van de Velde M. Nonobstetric Surgery during Pregnancy En: David H. Chestnut, Et al. Chestnut's Obstetric Anesthesia: Principles and Practice. 5ta ed. Filadelfia: Elsevier; 2014. p. 358-376.

10. Practice guidelines for obstetric anesthesia: An updated report by the American Society of Anesthesiologists Task Force on Obstetric Anesthesia. Anesthesiology 2016; 124:270-300.

11. Marino T, Park JS, Norwitz ER. Antepartum Fetal Assessment and Therapy. En David H. Chestnut, Et al Chestnut's Obstetric Anesthesia: Principles and Practice. 5ta ed. Filadelfia: Elsevier Inc; 2014. p. 95-122.

12. Mauricio Vasco Ramírez $\square$. Anestesia para cirugía fetal. Rev Colomb Anestesiol 2012; 40:268-72.

13. Anwari JS, Tareen Z. Anesthesia for fetoscopic intervention. Saudi Journal of Anaesthesia. 2014; 8(3):428-431.

14. Marc Van de Velde Frederik De Buck Fetal and Maternal Analgesia/Anesthesia for Fetal Procedures, Fetal Diagn Ther 2012; 31:201-209.

15. Ritu Gupta MB. Fetal surgery and anaesthetic implications. British Journal of Anaesthesia 2008; 8: 71-75.

16. Hans $P$, Sviggum MD, Bhavani SK. Maternal Anesthesia for Fetal Surgery. Clin Perinatol 2013; 40: 413-427. 